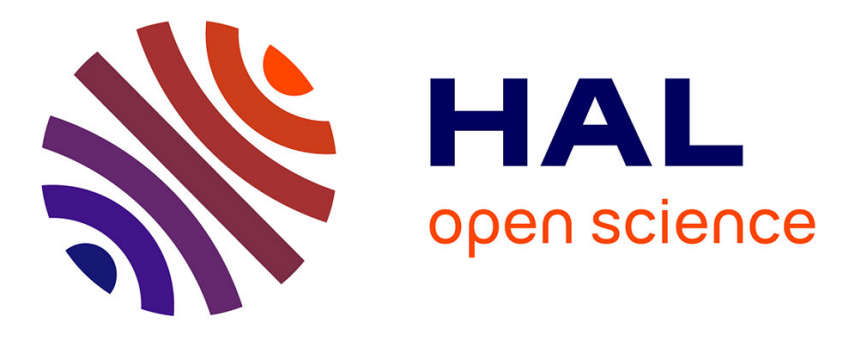

\title{
Detailed experimental and kinetic modeling study of 3-carene pyrolysis
}

Jia Zhang, Florence Vermeire, Ruben van de Vijver, Olivier Herbinet, Frederique Battin-Leclerc, Marie-françoise Reyniers, Kevin M van Geem

\section{To cite this version:}

Jia Zhang, Florence Vermeire, Ruben van de Vijver, Olivier Herbinet, Frederique Battin-Leclerc, et al.. Detailed experimental and kinetic modeling study of 3-carene pyrolysis. International Journal of Chemical Kinetics, 2020, 52, pp.785 - 795. 10.1002/kin.21400 . hal-03032789

\section{HAL Id: hal-03032789 \\ https://hal.science/hal-03032789}

Submitted on 16 Dec 2020

HAL is a multi-disciplinary open access archive for the deposit and dissemination of scientific research documents, whether they are published or not. The documents may come from teaching and research institutions in France or abroad, or from public or private research centers.
L'archive ouverte pluridisciplinaire $\mathbf{H A L}$, est destinée au dépôt et à la diffusion de documents scientifiques de niveau recherche, publiés ou non, émanant des établissements d'enseignement et de recherche français ou étrangers, des laboratoires publics ou privés. 


\title{
Detailed experimental and kinetic modeling study of 3-carene pyrolysis
}

\author{
Jia Zhang1, Florence Vermeire ${ }^{1}$, Ruben Van de Vijver ${ }^{1}$, Olivier Herbinet ${ }^{2}$, \\ Frédérique Battin-Leclerc ${ }^{2}$, Marie-Françoise Reyniers ${ }^{1}$, Kevin M. Van Geem ${ }^{1}{ }^{*}$ \\ 1 Laboratory for Chemical Technology, Ghent University, Gent, Belgium \\ 2 Université de Lorraine, CNRS, LRGP, Nancy, France
}

\begin{abstract}
Published in International Journal of Chemical Kinetics (2020), 52, 785-795. http://dx.doi.org/10.1002/kin.21400
\end{abstract}

\begin{abstract}
3-Carene is an important potential biofuel with properties similar to the jet-propellant JP-10. Its thermal decomposition and combustion behavior is to date unknown, which is essential to assess its quality as a fuel. A combined experimental and kinetic modeling study has been conducted to understand the initial decomposition of 3-carene. The pyrolysis of 3-carene was investigated in a jet-stirred quartz reactor at atmospheric pressure, at temperatures varying from 650 to $1050 \mathrm{~K}$, covering the complete conversion range. The decomposition of 3-carene was observed to start around $800 \mathrm{~K}$, and it is almost complete at $970 \mathrm{~K}$. Online gas chromatography shows that primarily aromatics are generated which suggests that 3-carene is not a good fuel candidate. The potential energy surface for the initial decomposition pathways determined by KinBot shows that a hydrogen elimination reaction dominates, giving primarily cara-2,4-diene. Next to this molecular pathway, radical pathways lead to aromatics via ring opening. The kinetic model was automatically generated with Genesys and consists of 2565 species and 9331 reactions. New quantum chemical calculations at the CBS-QB3 level of theory were needed to calculate rate coefficients and thermodynamic properties relevant for the primary decomposition of 3-carene. Both the conversion of 3-carene and the yields of the primary products (ie, benzene and hydrogen gas) are well predicted with this kinetic model. Rate of production analyses shows that the dominant pathways to convert 3-carene are hydrogen elimination reaction and radical chemistry.
\end{abstract}

Keywords: 3-carene, aromatics formation, automatic kinetic model generation, jet-stirred reactor, potential energy surface

\section{*Corresponding author information:}

Kevin M. Van Geem, Laboratory for Chemical Technology, Ghent University, Technologiepark 125, 9052 Gent, Belgium. Email: kevin.vangeem@ugent.be 


\section{Introduction}

Traditional fossil resources are nonrenewable and contribute to the emission of greenhouse gasses and particulate matter which raises environmental concern and makes it urgent to find alternative energy resources, such as biomass. To date, there is no biomass-derived alternative to replace current rocket fuels such as JP-10 with derived cetane number of 20.4. ${ }^{1}$ Terpenes, and in particular 3-carene, could be interesting alternatives for these high-energy density fuels. For example, Harvey ${ }^{2}$ demonstrated that a series of terpene fuel blends were proven to increase the cetane number and energy density of the jet fuel. Terpenes are natural occurring molecules with a structure consisting of a number of isoprene units. They are widely used in perfumes and fragrances. Kulkarni et al. were the first to suggest 3-carene, more precisely a blend of $75 \mathrm{wt} \%$ ethylidenenorbornene and $25 \mathrm{wt} \% 3$-carene as a promising high-energy density fuel ${ }^{3}$. 3-Carene is a bicyclic monoterpene with the same molecular formula as JP-10, that is, $\mathrm{C}_{10} \mathrm{H}_{16}$, the structural formula of 3-carene is shown in Figure 1, and today it is primarily produced by extraction from plants such as grass (Pinuslongifolia), Zeravschaniamembranacea and Pinusroxburghii. ${ }^{4-8}$ Ignition delay experiments of a 3-carene $/ \mathrm{O}_{2} / \mathrm{Ar}$ mixture performed by Sharath et al. ${ }^{9}$ showed that 3 -carene indeed has potential as fuel and that the ignition delay time of 3-carene is lower than that of JP-10 under similar conditions.

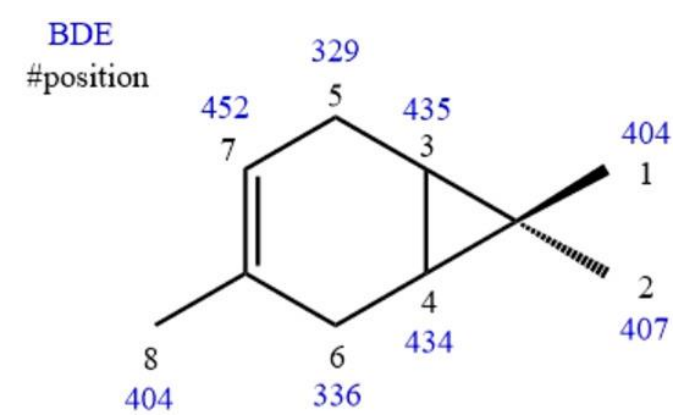

Figure 1: Structural formula of 3-carene along with $\mathrm{C}-\mathrm{H}$ bond dissociation energy $(\mathrm{kJ} / \mathrm{mol})$ calculated at the CBS-QB3 level of theory at $0 \mathrm{~K}$.

At present, the pyrolysis of 3-carene has not been extensively studied as opposed to its fossil alternative JP-10.10-15 Up to now, only two studies are reporting pyrolysis results of 3-carene. The first thermal decomposition study was done by Cocker et al., with a packed heated tube reactor using liquid 3-carene. ${ }^{16}$ They performed the experiments in a temperature range of 683-948 K with space time around $1 \mathrm{~min}$. The experimental pressure was not clearly mentioned in this paper. The decomposition was reported to start at $723 \mathrm{~K}$, and initially yields mainly $\mathrm{m}$ - and p-cymene. At higher temperatures, aromatics were also formed such as benzene and toluene. 3-Carene was fully converted at temperatures above $833 \mathrm{~K}$.

Sharath et al. ${ }^{17}$ report the second pyrolysis study of 3-carene in a reflected single pulse shock tube reactor. The dwell time in these experiments was around $1.5 \mathrm{~ms}$, and its operational pressure varies from 8 to $14 \mathrm{bar}$, with the temperature ranging from 920 to $1220 \mathrm{~K}$. Linear hydrocarbons such as acetylene were found to be the primary products. A kinetic model consisting of 44 reactions involving 37 species was obtained to describe the initial thermal decomposition of 3 -carene. The kinetic parameters were derived from transition state theory calculations and 
refined to fit the experimental data. This model is derived mainly for the prediction of acetylene, allene, butadiene, toluene, cyclopentadiene, and 3-carene, and it is also used to simulate the current set of experiments performed in this work.

No consensus exists about the product distribution during 3-carene pyrolysis. Cocker et al. ${ }^{16}$ mainly observed aromatic products, while Sharath et al. ${ }^{17}$ mainly observed linear hydrocarbons.

To gain a better understanding of the pyrolysis chemistry of 3-carene, this study reports a new and more detailed experimental and kinetic investigation of 3-carene pyrolysis. The experiments are performed in a quartz jet-stirred reactor with the temperature ranging from 650 to $1050 \mathrm{~K}$ and an average residence time of $2 \mathrm{~s}$. The products are identified and quantified with online gas chromatography and mass spectrometry. The experiments were conducted at atmospheric pressure that is complementary to the pressures used in previous 3-carene shock tube reactor experiments. Comparing with the existing model of Sharath et al.,17 which only considers small species, a more detailed and extensive model has been generated that also considers the formation of aromatics. The potential energy surface (PES) for the initial decomposition pathways is studied with the help of KinBot, which is an automatic PES exploration tool. ${ }^{18} \mathrm{~A}$ kinetic model for 3-carene initial decomposition was generated with the help of the in-house automatic kinetic model generation tool Genesys. ${ }^{19,20}$

\section{Experimental methods}

The initial purity of the 3-carene was $95.90 \mathrm{wt} \%$, which is not sufficiently high for the envisioned kinetic study. A comprehensive gas chromatograph (GC $\times \mathrm{GC}$ ) was used to identify the impurities, and its chromatogram is shown in Figure 2. The identified impurities are mainly mononaphthenes with one potential molecular structure indicated for each identified peak in the chromatogram. Before starting the experimental study of 3-carene pyrolysis, the 3-carene sample was purified through vacuum distillation with which the weight percentage of 3-carene increased from 95.90 wt $\%$ to $98.90 \mathrm{wt} \% . \mathrm{C}_{10} \mathrm{H}_{16}$ isomers are found to persist as impurities after the purification. The total weight percentage is $1.09 \mathrm{wt} \%$ and detailed feedstock composition before and after the purification can be found in the Supporting Information.

The pyrolysis of 3-carene is studied in an isothermal quartz jet-stirred reactor. The experimental setup has been described in detail by Herbinet and Battin-Leclerc. ${ }^{21}$ The main features of the apparatus and analytical equipment are given below in Figure 3. 3-Carene is heated in the evaporator prior to mixing with inert helium (Messer; 99.99\%). The mixture is subsequently fed to the reactor though an annular preheating zone. The preheating section is split into two temperature zones. The temperature in the second zone is set to the reactor temperature. This guarantees that the mixed gas is heated to the reactor temperature before flowing into the reactor. The reactivity of 3-carene in this section is negligible because the residence time in the preheating section is only a few percent of the average residence time in the reactor. ${ }^{22}$ The heated and wellmixed flow is injected into the jet-stirred reactor. This is equipped with a K-type thermocouple used to measure and control the reactor gas temperature. The reactor pressure is set with a needle valve downstream of the reactor. 


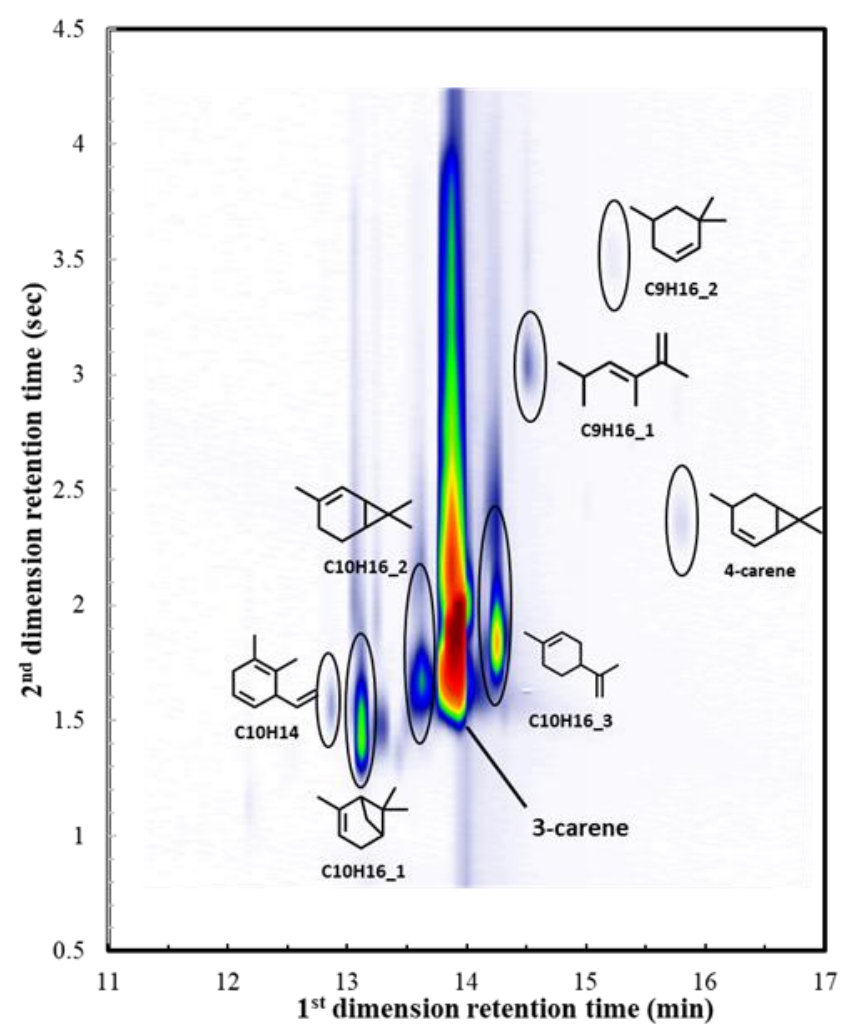

Figure 2: The two-dimensional gas chromatogram result for the 3-carene analysis before the purification process. One potential molecular structure is given for each indicated peak in the gas chromatogram.

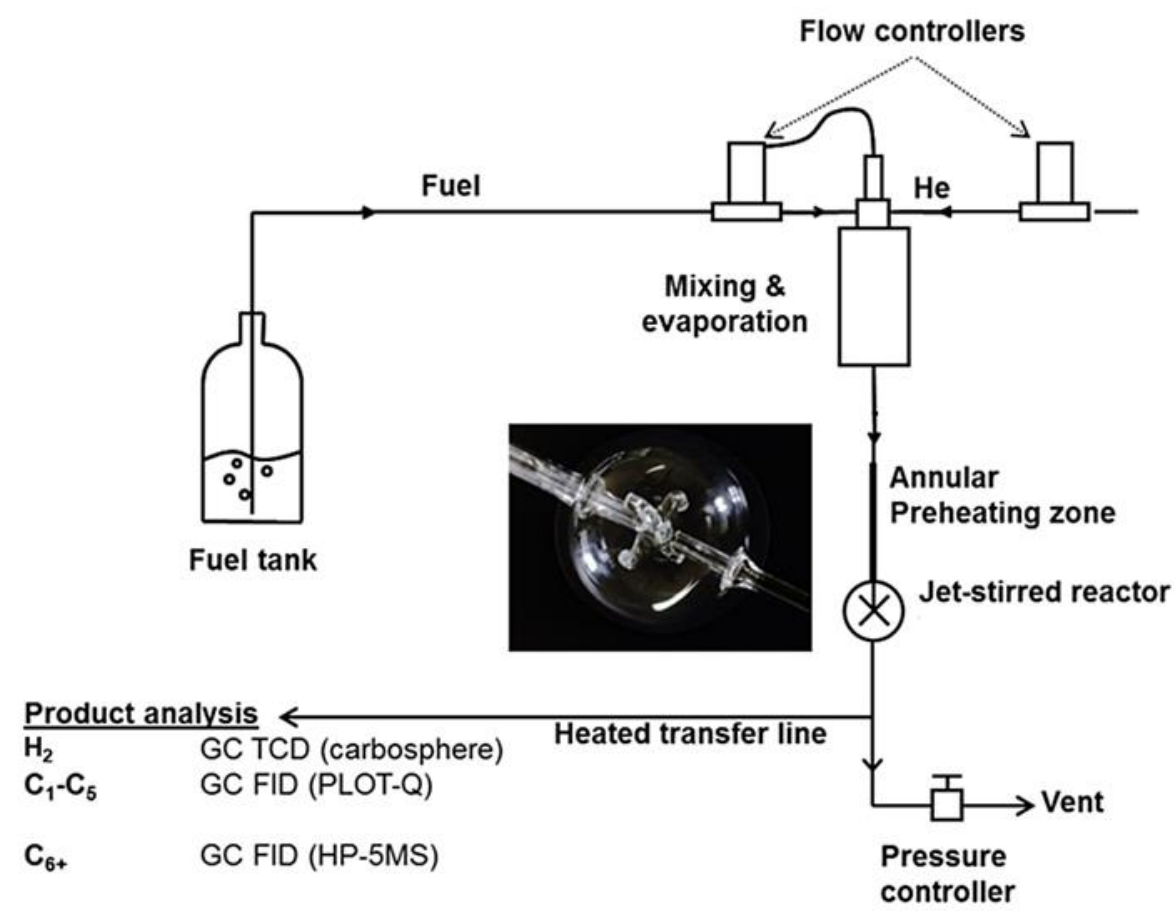

Figure 3: Schematic representation of the jet-stirred reactor setup indicating the most important flow/pressure controllers and the implemented analytic tools. The picture of the reactor is also shown with the cross-shaped nozzle seen in the center of the reactor. 
The quartz reactor volume is $81.2 \mathrm{~cm}^{3}$. The temperature ranges from 650 to $1050 \mathrm{~K}$, and the pressure is set to $1.07 \mathrm{bar}$ for all experiments. The volumetric flow rate calculated with the inlet flow rate and experimental condition ( $\mathrm{T}, \mathrm{P}$ ) is set to $40.6 \mathrm{~cm}^{3} \mathrm{~s}^{-1}$, which leads to an average residence time of $2.0 \mathrm{~s}$. The inlet mole fraction of 3 -carene is 0.025 .

The effluent of the reactor was injected into three gas chromatographs (GCs) that allow online quantification of product species. The transfer line between the reactor outlet and GC is heated to $433 \mathrm{~K}$ to avoid condensation. The first GC is equipped with a Carbosphere packed column and a thermal conductivity detector. This GC is used to detect hydrogen gas with argon (Ar) as the carrier gas. A PLOT-Q capillary column and a flame ionization detector (FID) preceded by a methanizer are installed after the second GC. Compounds with two carbon atoms up to five carbon atoms can be accurately identified by this GC. The outlet flow is also analyzed with a third GC equipped with a HP-5 ms capillary column and FID. Product identification is done offline with a fourth a GC connected to a quadrupole mass spectrometer (GC-MS). The GC can be equipped with PLOT-Q or HP-5 ms column. The mass range of the MS is from 10 to $400 \mathrm{~m} / \mathrm{z}$.

Products are calibrated by either injecting a known amount of the species in the GC or by using the effective carbon number method. If the first method is used, the relative experimental error equals $5 \%$ based on experience. The latter is done for 3-carene and for hydrocarbons with up to four heavy atoms. If calibration is done with the effective carbon number method, the relative experimental error is based on previous work set to $10 \%$. The carbon balances are closed within $10 \%$.

\section{Computational methods}

The PES for initial decomposition of 3-carene was explored using KinBot, ${ }^{18}$ which is designed to automatically crawl on PESs to find all kinetically relevant stationary points. It starts from 3-carene to search for connected transition states, from which it identifies product species with Intrinsic Reaction Coordinate calculations.

For the resulted species and reactions found by KinBot, and other important decomposition species and reactions, thermodynamic properties and rate coefficients were calculated from first principles. Both for the species and the transition states, the lowest energy conformers were identified using the B3LYP/6-31G (d) level of theory. For the lowest energy conformers, CBS-QB323 calculations were done to obtain the electronic energy and the frequencies. Internal rotations were treated using one-dimensional hindered rotations (1D-HIR). The hindrance potentials were calculated at the B3LYP/6-31G(d) level of theory with relaxed surface scans. Thermodynamic properties were calculated using statistical thermodynamics, and the reaction rate coefficients were calculated through classical transition state theory. More information on this can be found in Van de Vijver et al. ${ }^{24}$ To facilitate the CHEMKIN simulation, the species thermodynamics are represented in NASA polynomials, and the rate coefficients are regressed using simple or modified Arrhenius expressions.

All electronic structure calculations were performed on the high-performance supercomputer at Ghent University using Gaussian $16 .{ }^{25}$ All the calculated thermodynamic data and reaction rate 
coefficients along with the optimized electronic structures are given in the Supporting Information.

\section{Kinetic model development}

A new elementary step kinetic model has been developed to describe the initial decomposition of 3 -carene. The model was developed automatically by Genesys, ${ }^{19}$ a rule-based network generation tool. This means that species and reactions are only added to the model if they meet user-defined constraints. These constraints are used to either limit the applicability of a reaction family by constraining the possible reactant structure, or to limit product formation by general product constraints, applicable on all species in the model. The obtained thermodynamics and kinetics from quantum chemistry were included into the Genesys databases which acts as the thermodynamic and kinetic source during the kinetic model construction. ${ }^{19,20}$ A general assessment of the uncertainty on theoretical calculations with the method used in this work is included. This is made based on previous studies using the same methodology. The core of the kinetic model (ie, the thermochemical and kinetic data) is automatically calculated in Genesys, by the methods given below. The full mechanism of 2565 species and 9331 reactions was automatically generated to describe the 3-carene pyrolysis. No rate constant was tuned in this model to meet the experimental results. The developed kinetic model of 3-carene pyrolysis will be used to perform the reactor simulations using the continuously stirred tank reactor model in CHEMKIN 26 to test the model performance. This kinetic model can be found in the Supporting Information in the CHEMKIN input format.

\subsection{Thermochemistry}

For each species in the kinetic model, Genesys calculates the standard enthalpy of formation, the standard entropy and the heat capacity. Genesys uses two databases for this. The first database contains thermodynamic values from experimental data or high-level ab initio calculations for a set of species. Most data in Genesys originate from CBS-QB3 calculations as described in the Computational Methods section. If a species from the model is present in that database, these values can be used as is. Sabbe et al. ${ }^{27}$ evaluated the uncertainty of the standard enthalpy of formation of hydrocarbons and hydrocarbon radicals by comparing with the experimental data. By involving the bond additive corrections, the mean absolute deviation is found to be better than $2 \mathrm{~kJ} / \mathrm{mol}$. When a species is absent from the database, Genesys relies on Benson's group additivity method 28 and Lay's hydrogen bond increment method. ${ }^{29}$ Additionally, ring strain corrections (RSC) and non-next-nearest neighbor interactions are introduced to account for the nonbonded interaction in the species thermodynamics calculation. The RSCs play an important role in this case because cyclic components account for a big portion of the experimental products. A specific RSC is required for each type of ring with different ring size and number of endocyclic double bonds. Some specified RSCs were determined by several groups. ${ }^{27,30}$ The group additivity values and hydrogen bond increments used in this work are those calculated by Sabbe et al.27,31 


\subsection{Kinetics}

For the kinetics, a similar two-database approach is used. There is a database with ab initio results for several reactions, and if a reaction is not found in that database, the kinetics are approximated with the group additivity method developed by Saeys et al. ${ }^{32}$ and extended by Sabbe et al. ${ }^{33}$ The ab initio kinetics are calculated at CBS-QB3 level of theory combined with 1D-HIR corrections and Eckart tunneling. ${ }^{34}$ The uncertainty of the rate parameters between 600 and $1000 \mathrm{~K}$ is below a factor of 4. Several rate coefficient uncertainty studies were done on hydrocarbon-related reactions at CBS-QB3 level of theory. Sabbe et al. studied the hydrogen radical ${ }^{11}$ and carboncentered radical ${ }^{7}$ addition and $\beta$-scission reactions. The deviation between the theoretical and experimental rate coefficients is within a factor of 2 and 3 , respectively. The hydrogen abstraction reactions were investigated by Saeys et al. ${ }^{35}$ and Sabbe et al, ${ }^{36}$ and the deviation is found to be within a factor of 2-4. Based on these studies, the uncertainty on the kinetic data determined at the CBS-QB3 level of theory is assumed to be within a factor 2-4. For hydrogen abstraction reactions, the group additivity values determined by Sabbe et al. ${ }^{36}$ are used. For the intramolecular hydrogen abstraction reaction, the group additivity value comes from Van de Vijver et al. ${ }^{37}$ For addition reactions, group additivity values are obtained from Sabbe et al. ${ }^{33,38}$ For several reactions during 3-carene pyrolysis, no reaction rate coefficients are available, and no corresponding group additivity values either. For these reactions, rate rules were developed based on known reactions with similar reactants. Three important reaction families for which rate rules are used as follows: (1) hydrogen radical addition reactions on aromatics, (2) hydrogen gas elimination reactions, and (3) Diels-Alder reactions. For reaction family (1), the hydrogen radical addition reaction on benzene is used, for family (2) the hydrogen gas elimination reaction from 1,4-cyclohexadiene to benzene is used, and for family (3) the Diels-Alder reaction on 1,3-butadiene and ethylene is used.

\section{Results and discussion}

\subsection{Potential energy surface explored via KinBot}

The initial decomposition pathways for 3-carene pyrolysis were explored by KinBot and the PES is shown in Figure 4, which reports the electronic energies of the various stationary points.

There are three different reaction types observed in the initial decomposition stage of which the hydrogen gas elimination reaction has the lowest energy barrier. This reaction proceeds by the formation of a hydrogen gas molecule from the hydrogen atoms on positions 5 and 6 (Figure 1) forming cara-2,4-diene. Note that this reaction can proceed through two separate transition states, which differ by the position of the hydrogen atoms relative to the position of the threemembered ring. In the transition state with the lowest energy, the hydrogen atoms forming hydrogen gas are positioned on the opposite of the three-membered ring relative to the plane of the six-membered ring. Note that this reaction is similar to the hydrogen elimination reaction on cyclohexene and 1,4-cyclohexadiene. Their activation energy is 298 and $180 \mathrm{~kJ} / \mathrm{mol}$, respectively, in the NIST Chemical Database. The optimized geometries of this PES are all given in Supporting Information. A Diels-Alder reaction producing isoprene and 3,3-dimethyl-cyclopropene directly from 3-carene is another important pathway, with 3,3-dimethyl-cyclopropene that can further lead to isoprene via an isomerization reaction. Apart from these two reactions, several reactions 
opening the three-membered ring were identified by KinBot. These reactions simultaneously open the three-membered ring and move a hydrogen atom to form carbene intermediates. The latter subsequently isomerize to stable products via a second hydrogen shift.

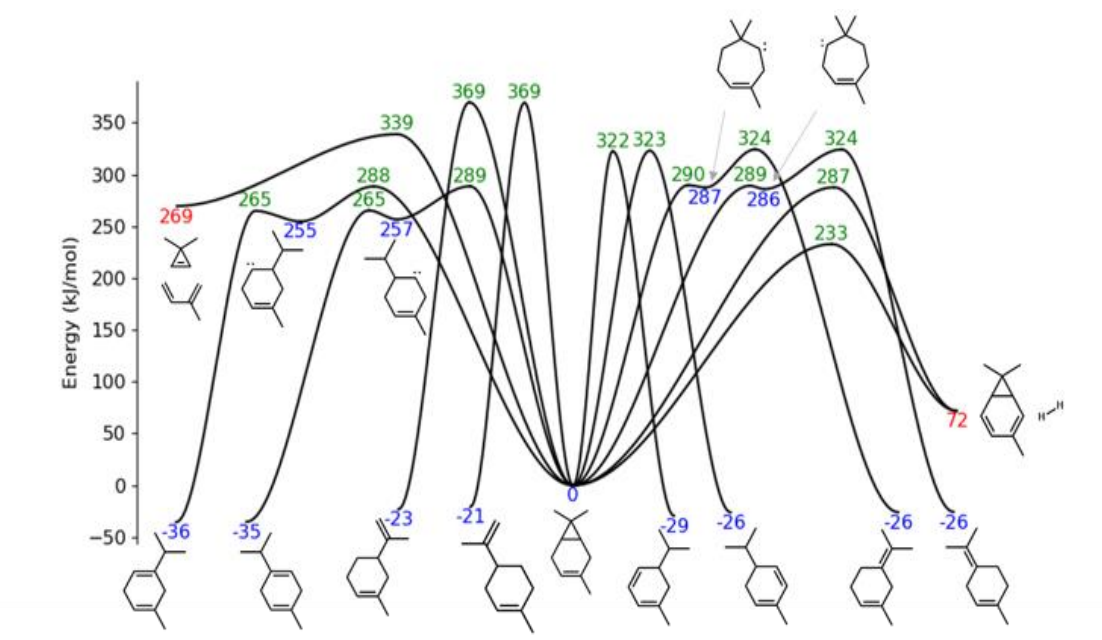

Figure 4: The electronic PES for the initial decomposition of 3-carene using KinBot. The electronic energy was calculated at CBS-QB3 level of theory. The values in blue correspond to wells on the PES, values in red are bimolecular products, and values in green are transition state energies.

\subsection{Experimental results and model simulation}

The online GCs are employed to quantify the effluent composition. About hundred species were identified, of which several major products were detected on each of the two GCs used for quantification. The detailed experimental results for the main products can be found in the Supporting Information. The mole fractions of the most important primary products from the 3carene pyrolysis experiments are depicted in Figures 5 and 6. Aromatics and low molecular weight products (C5-) such as hydrogen gas, methane, and ethylene are observed as major products. The decomposition of 3-carene starts at $800 \mathrm{~K}$, and 3-carene is completely converted at $970 \mathrm{~K}$ for the studied residence time. The experimental observed decomposition temperature is higher than that of the previous heated packed-tube reactor experiments. ${ }^{16}$ This results from the effect of experimental operation conditions and dilution. The mole fractions of $\mathrm{p}$-cymene and $\mathrm{m}$ cymene show a peak around $900 \mathrm{~K}$, while the peak for the mole fraction of p-cymenene is around $950 \mathrm{~K}$. The same trend was also observed for propylene, and its peak is found around $950 \mathrm{~K}$. An increasing mole fraction as function of the conversion was observed for the products such as styrene, toluene, benzene, acetylene, ethylene, methane, and hydrogen. Methane and hydrogen gas are observed to have the highest mole fraction among these products. The detailed experimental results and product compositions can be found in the Supporting Information. 

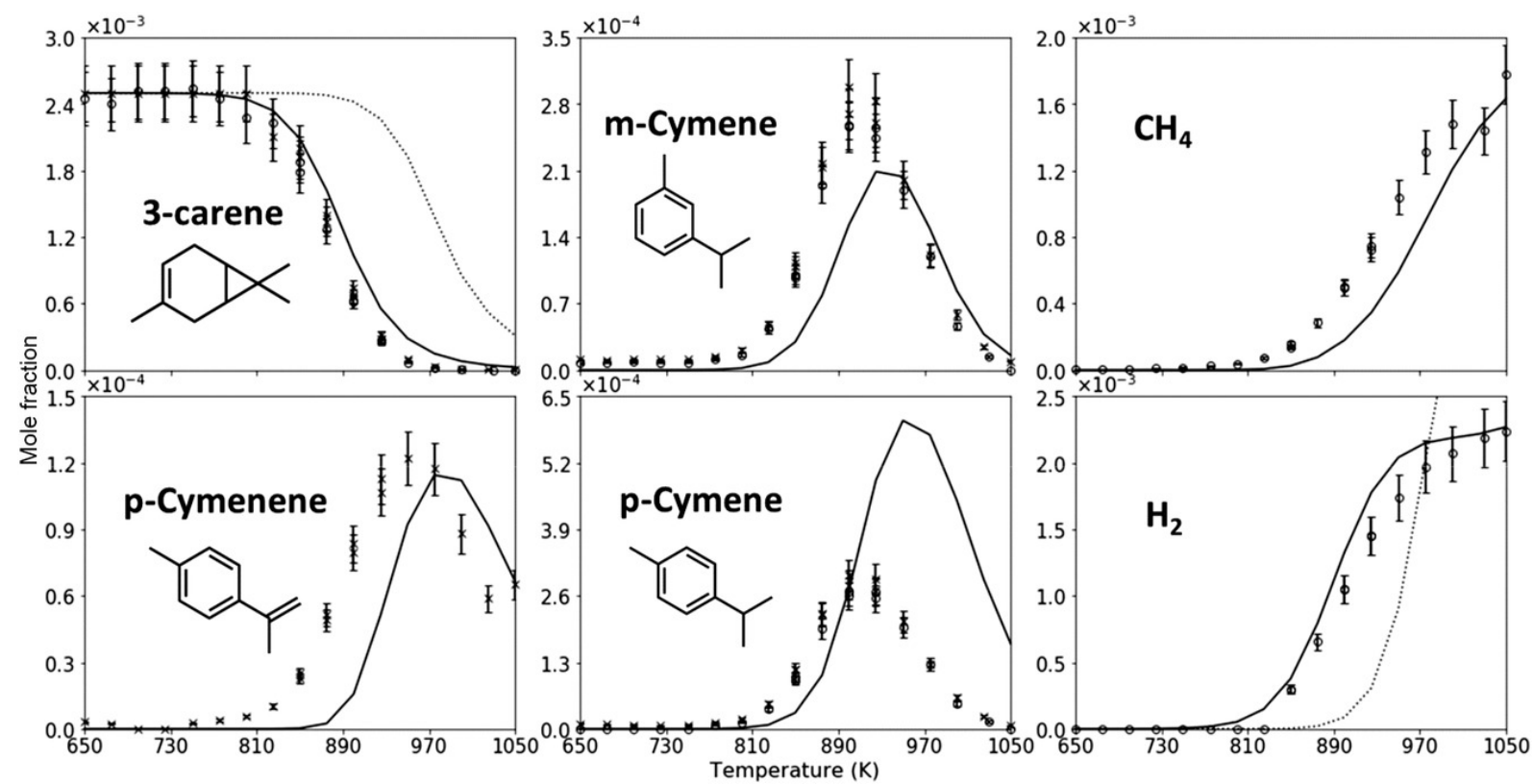

Figure 5: Species mole fractions as a function of temperature for the pyrolysis of 3-carene.

Experiments (points: GC with Q-bond capillary column, cross: GC with HP-5 ms capillary column) and simulation results (solid line: new developed kinetic model, dotted line: Sharath et al. kinetic model ${ }^{17}$ ) are compared. Experimental conditions are 1.07 bar, $2.0 \mathrm{~s}$ average residence time, and inlet mole fraction of 3-carene is $2.5 \times 10^{-3}$. Simulations are performed with CHEMKIN software using the continuous stirred-tank reactor model.
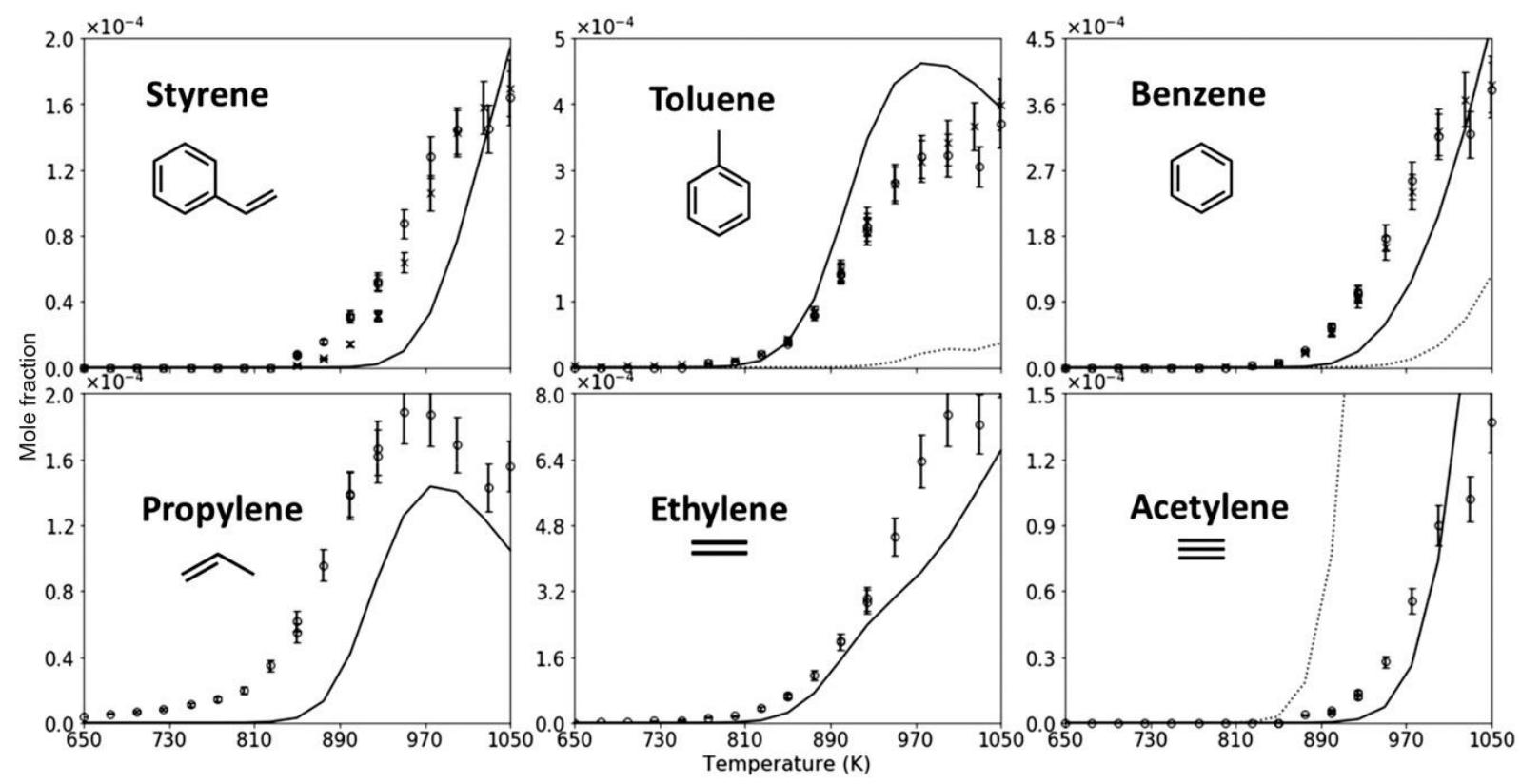

Figure 6: Species mole fractions as a function of temperature for the pyrolysis of 3-carene.

Experiments (points: GC with Q-bond capillary column, cross: GC with HP-5 ms capillary column) and simulation results (solid line: new developed kinetic model, dotted line: Sharath et al kinetic model ${ }^{17}$ ) are compared. Experimental conditions are 1.07 bar, $2.0 \mathrm{~s}$ average residence time, and inlet mole fraction of 3 -carene is $2.5 \times 10^{-3}$. Simulations are performed with CHEMKIN software using the continuous stirred-tank reactor model. 
The comparison between the simulation results and experimental data is shown in Figures 5 and 6. The mole fraction of most products can be well predicted by this kinetic model. The predicted mole fraction profile of 3-carene agrees well with the experimental results. The mole fraction profile of m-cymene and p-cymenene are well captured by the current model. m-Cymenene is also predicted by this model with a similar trend as p-cymenene, while this molecule is not detected experimentally. The general trend of p-cymene mole fraction profile is predicted by this model, however it is overpredicted at high temperature ( $>900 \mathrm{~K})$. For the aromatics formation, the model performance for styrene and benzene is good, while it slightly overpredicts the mole fraction of toluene at high temperature $(>900 \mathrm{~K})$. Ethylbenzene is also generated by this model of which the mole fraction profile trend is captured. For the low molecular weight products, this model is able to capture the trend for their mole fraction profiles. The mole fraction of methane, the major product from radical chemistry, is well predicted by the current model. Good agreements between experiments and simulations on the mole fraction profiles are also found for hydrogen gas, acetylene, ethylene, and propylene.

The sensitivity analysis study is performed at $900,950,1000$, and $1050 \mathrm{~K}$, and the corresponding conversion of 3 -carene is $59 \%, 89 \%, 97 \%$, and $99 \%$ to identify which reaction rate coefficients could make the model performance even better. Normalized sensitivity coefficients of the main reactions for the mole fractions of 3-carene, p-cymene, m-cymene, and toluene are given in the Supporting Information.

The model performance of Sharath et al. ${ }^{17}$ is also tested against experiments in this study. The simulated mole fraction profiles are indicated with the dotted line in Figures 5 and 6. This model is able to predict part of the mole fractions for experimental products, namely 3-carene, toluene, benzene, acetylene, and hydrogen gas. The other components were not considered in this model. An obvious temperature shift $(\sim 120 \mathrm{~K})$ can be observed in the mole fraction profile of 3-carene, which is indicated with the dotted line in Figure 5. Except for the overprediction at high temperature $(>950 \mathrm{~K})$, its behavior on the prediction of hydrogen gas is good. However, the mole fraction of acetylene is overpredicted through the entire experimental temperature range. This model is unable to predict the mole fraction of the aromatics. For example, aromatics with 10 carbon atoms are not even considered, while simple aromatics (i.e., benzene and toluene) are underpredicted compared with the experiments in this study. The other products simulated yields can be found in the Supporting Information. This model tends to overpredict the mole fraction of small molecules such as isoprene.

\subsection{Reaction path analysis}

\subsubsection{Initial decomposition pathways}

The primary 3-carene decomposition reaction pathways are depicted in Figure 7. A rate of production analysis was conducted at 850,950 , and $1050 \mathrm{~K}$ to study the relative importance of these pathways. The corresponding 3 -carene conversion is $17 \%, 89 \%$, and $99 \%$. In general, 3 -carene is consumed through hydrogen elimination reaction and hydrogen abstraction reactions. 


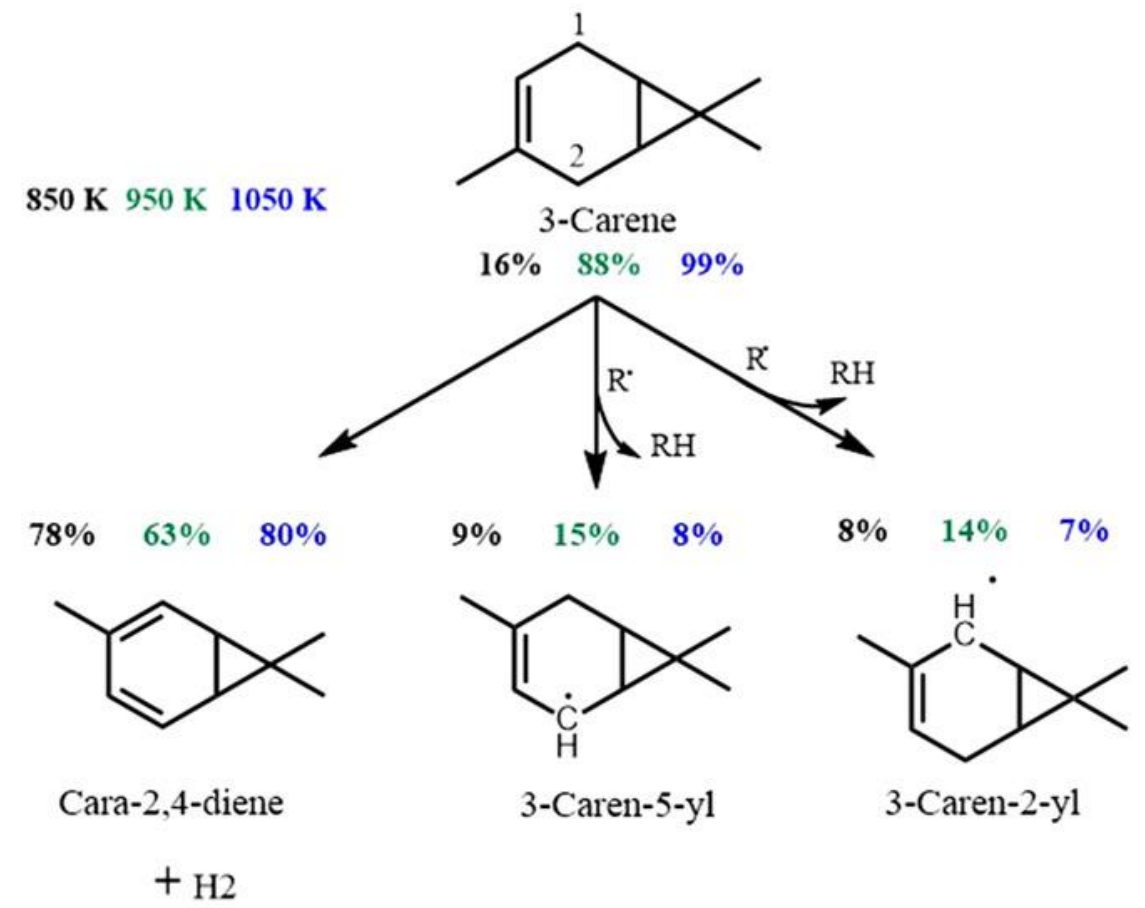

Figure 7: The initial decomposition reaction pathways for 3-carene pyrolysis at 850, 950, and $1050 \mathrm{~K}$ with relative rate of production (\%) indicated. Three rates of production values are calculated relative to the total consumption of 3-carene at the corresponding temperature. Their corresponding 3-carene conversion is also presented. $\mathrm{R} \bullet$ stands for the hydrogen abstracting radicals, which are mainly hydrogen radicals, methyl radicals, and 4-isopropylbenzyl radicals.

Most of 3-carene is consumed via the hydrogen elimination reaction which has the lowest activation energy among all the potential pathways. Hydrogen elimination reactions of 3-carene contributes the most for hydrogen gas formation. The radicals generated through hydrogen abstraction reactions on 3-carene can further react through C-C $\beta$-scission reactions opening the three-membered ring. These subsequent reactions will be discussed further on. In this study, except for hydrogen radicals and methyl radicals, the 4-isopropylbenzyl radical is another important hydrogen abstracting radical. Its dominant formation pathway is depicted in Figure 8. Cara-2,4-diene, the product formed by the hydrogen elimination reaction, is abstracted by the radical which produces the resonantly stabilized radical. This radical can react through $\mathrm{C}-\mathrm{C}$ $\beta$-scissions generating R1. Intramolecular hydrogen abstractions will occur on R1, which gives the more stabilized 4-isopropylbenzyl radical. All the involved reactions have a relatively high reaction rate, which leads to the accumulation of this radical. This radical will be converted into p-cymene through a hydrogen abstraction reaction, which leads to an overprediction of p-cymene at high temperature $(>900 \mathrm{~K})$. As the temperature increases, the radical chemistry reaction pathways start to gain importance. The absolute reaction rate of the radical chemistry pathway is the highest at about $900 \mathrm{~K}$, which is same as that for p-cymene and m-cymene mole 

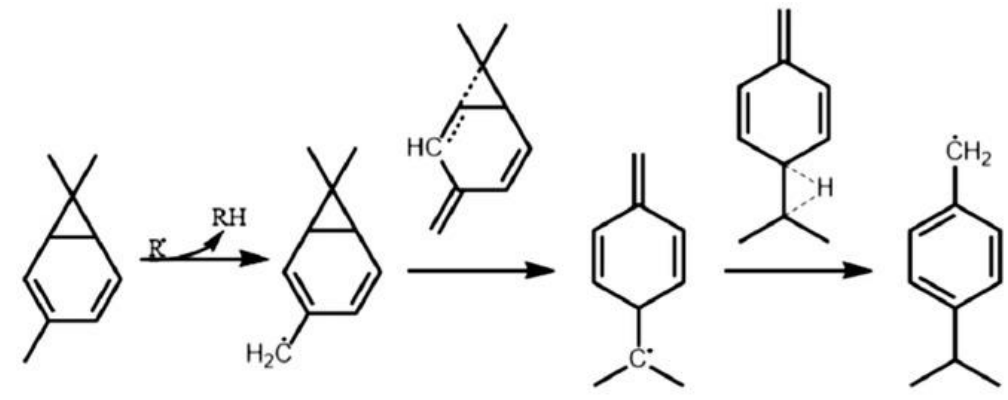

RI

Figure 8: Reaction pathway generating the 4-isopropylbenzyl, which is one of the important hydrogen abstracting radicals. The transition state for the $\mathrm{C}-\mathrm{C} \beta$-scission reaction and intramolecular hydrogen abstraction reaction are also indicated

\subsubsection{Secondary pathways}

A more detailed illustration of the most important decomposition pathways, which include the most important 3-carene consumption pathways, is depicted in Figure 9. The rate of production analysis is conducted at $950 \mathrm{~K}$. The channels for which the rate of production is smaller than $1 \%$ are not shown. At this temperature, the model predicts a 3-carene conversion of $89 \%$. Only the reactions with 1,4-substitution on the six-membered ring are shown, equivalent reactions with 1,3-substituted six-membered rings have similar rates of production. For example, the hydrogen abstraction reaction on 3-carene can generate either 3-caren-5-yl or 3-caren-2-yl. These two radicals can both react through $\mathrm{C}-\mathrm{C} \beta$-scission reactions opening the unstable three-membered ring. For simplicity, only the reaction pathway generating 1,5-p-menthadien-8-yl is shown in the detailed reaction network.

The radical chemistry starts with the hydrogen abstraction reaction, which generates 3-caren-2-yl radical. This product is favored due to the fact that $\mathrm{C}-\mathrm{H}$ bond is weaker in the position 5,6 as shown in Figure 1. C-C $\beta$-scissions lead to the 1,5-p-menthadien-8-yl radical. The latter can be converted via an intramolecular hydrogen abstraction reaction producing resonantly stabilized radicals. There are two potential positions available for this reaction. Part of the 1,5-p-menthadien-8-yl tends to react through $\mathrm{C}$-C $\beta$-scission reactions, eventually generating toluene and isopropyl radical. The isopropyl radical can be further converted to ethylene, which is one of the small molecules observed in the experiments. The generated toluene contributes to the generation of aromatics such as xylene and benzene. The other part of 1,5-p-menthadien-8-yl is converted to 1,5-p-menthadien-4-yl which generates p-cymene through $\mathrm{C}$ - $\mathrm{H} \beta$-scission reactions. p-Cymene mainly reacts via radical reactions (ie, hydrogen abstraction), which produce other observed aromatics such as styrene.

Mainly two types of reactions help to decompose cara-2,4-diene: (1) Hydrogen abstraction reactions and (2) carbene type reactions which open the three-membered ring. Figure 9 shows that the most of the generated cara-2,4-diene further reacts through hydrogen abstraction reactions. Four positions (Figure 9) are dominant for hydrogen abstraction reactions. In this figure, the abstraction reaction on position 3 is not shown, which is similar to that for position 2 . The subsequent reactions related to 2,4-caradien-10-yl can be found in Figure 8, which is also not shown in this detailed reaction network. 2,4-caradien-6-yl reacts through the $C$ - $C \beta$-scission 
reaction in which the three-membered ring will open. It generates the same product (p-cymen-8-yl) as the hydrogen abstraction on p-cymene. A similar ring-opening reaction is also observed for 2,4-caradien-8-yl, which produces the R2 and R3. These two radicals produce toluene and 1-propen-2-yl through the $\mathrm{C}$-C $\beta$-scission reaction. The generated 1-propen-2-yl will further abstract hydrogen atoms generating propylene.

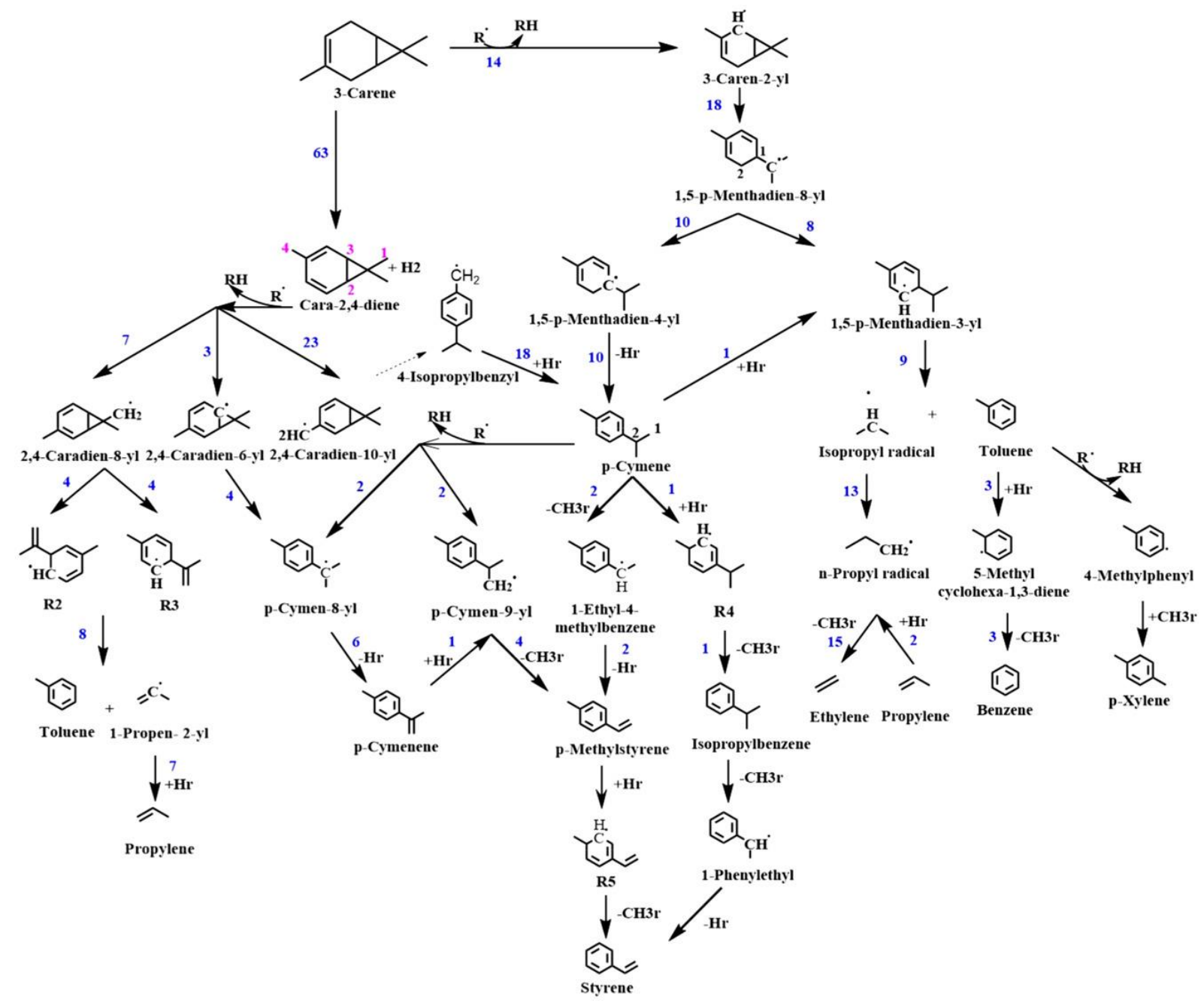

Figure 9: The detailed reaction network with rate of production values for the two dominating reaction pathways (molecular and radical chemistry) for 3-carene pyrolysis. Rate of production values, calculated relative to the total consumption of 3-carene, are presented at $950 \mathrm{~K}$. R stands for the hydrogen abstracting radicals, which are mainly hydrogen radicals, methyl radicals, and

4-isopropylbenzyl radicals. 3-Caren-2-yl is taken as an example to represent the products generated from the hydrogen abstraction reaction from 3-carene. The dashed arrow represents the reaction pathway related to the $\mathrm{R} 2$ with some intermediates ignored to make the reaction network easy to read. 


\section{Conclusions}

The chemistry of the thermal decomposition of 3-carene is studied experimentally and theoretically. A new experimental dataset is gathered in a jet-stirred reactor under atmospheric pressure and temperatures ranging from 650 and $1050 \mathrm{~K}$. The experimental results show that the major products are aromatics and low molecular weight molecules such as methane. The kinetics and thermodynamics for the initial decomposition chemistry were calculated at the CBS-QB3 level of theory. A kinetic model was automatically generated by Genesys, which consisted of 2565 species and 9331 reactions. The experimental conversion of 3-carene is accurately predicted with this kinetic model considering that no rate constant was tuned to match the experimental data. Apart from the hydrogen elimination reaction, the decomposition chemistry is dominated by radical reactions. The pathways to the aromatic products such as benzene have been identified and the kinetic model is able to grasp the general trend for their formation. This work shows that Genesys is capable to automatically generate a kinetic model for complex molecules considering among others the aromaticity generation pathways. The new kinetic model is thus the first model that describes the 3-carene pyrolysis in all its detail, which will serve as basis for other terpenes and oxidation studies of 3-carene and related compounds.

\section{Acknowledgments}

This work was carried out using the STEVIN Supercomputer Infrastructure at Ghent University, funded by Ghent University, the Flemish Supercomputer Center (VSC), the Hercules Foundation and the Flemish Government - department EWI. The authors would like to acknowledge the financial support from the China Scholarship Council (CSC), the SBO proposals "Bioleum" and "Arboref" supported by the Institute for promotion of Innovation through Science and Technology in Flanders (IWT), the Fund for Scientific Research Flanders (FWO), and the European Research Council under the European Union's Horizon 2020 research, and innovation programme/ERC grant agreement $n^{\circ}$ 818607. Ruben Van de Vijver acknowledges financial support from a postdoctoral fellowship from the Research Foundation - Flanders (FWO) grant number 3E013419.

\section{Supporting Information}

- A word file with supplementary material

- An excel spreadsheet with experimental data

- A text file containing the model 


\section{References}

1 Harvey BG, Merriman WW, Koontz TA. High-density renewable diesel and jet fuels prepared from multicyclic sesquiterpanes and a 1-hexene-derived synthetic paraffinic kerosene. Energy Fuels. 2015; 29: 2431- 2436.

2 Harvey BG, Ridgecrest C. High density turbine and diesel fuels from tricyclic sesquiterpenes, US Patent No. 9,777,234, Washington, DC; 2018.

3 Kulkarni SG, Bagalkote VS, Patil SS, Kumar UP, Kumar VA. Theoretical evaluation and experimental validation of performance parameters of new hypergolic liquid fuel blends with red fuming nitric acid as oxidizer. Propellants, Explos Pyrotech. 2009; 34: 520- 525.

4 Simonsen JL. The constituents of Indian turpentine from Pinus longifolia, Roxb. Part I. J Chem Soc Trans. 1920; 117: 570- 578.

5 Simonsen JL. The constitution of the terpene present in the essential oil from Andropogon Jwarancusa, Jones. J Chem Soc Trans. 1922; 121: 2292- 2299.

6 Simonsen JL, Rau MG. The constituents of Indian turpentine from Pinus longifolia , roxb. Part II. J Chem Soc Trans. 1923; 123: 549- 560.

7 Saei-Dehkordi SS, Khalighi-Sigaroodi F, Pirali-Kheirabadi K, et al. Chemical composition, antioxidative capacity and antimicrobial activity of Z eravschania membranacea (B oiss.) P imenov essential oil. J Food Saf. 2014; 34: 87- 94.

8 Salem MZM, Ali HM, Basalah MO. Essential oils from wood, bark, and needles of Pinus roxburghii Sarg. from Alexandria, Egypt: antibacterial and antioxidant activities. BioResources. 2014; 9: 7454- 7466.

9 Sharath N, Reddy K, Barhai P, Arunan E. Ignition delay of 3-carene: single pulse shock tube study. Curr Sci. 2015; 108: 2083.

10 Chenoweth K, van Duin ACT, Dasgupta S, Goddard Iii WA. Initiation mechanisms and kinetics of pyrolysis and combustion of JP-10 hydrocarbon jet fuel. J Phys Chem A. 2009; 113: 1740- 1746.

11 Guo F, Cheng X. ReaxFF molecular dynamics study of initial mechanism of JP-10 combustion. Combust Sci Technol. 2012; 184: 1233- 1243.

12 Magoon GR, Aguilera-Iparraguirre J, Green WH, et al. Detailed chemical kinetic modeling of JP-10 (exotetrahydrodicyclopentadiene) high-temperature oxidation: exploring the role of biradical species in initial decomposition steps. Int J Chem Kinet. 2012; 44: 179- 193.

13 Gao CW, Vandeputte AG, Yee NW, et al. JP-10 combustion studied with shock tube experiments and modeled with automatic reaction mechanism generation. Combust Flame. 2015; 162: 3115- 3129.

14 Zhao L, Yang T, Kaiser RI, et al. A vacuum ultraviolet photoionization study on high-temperature decomposition of JP-10 (Exo-Tetrahydrodicyclopentadiene). PCCP. 2017; 19: 15780- 15807.

15 Morozov A, Mebel A, Kaiser R. A theoretical study of pyrolysis of exo-tetrahydrodicyclopentadiene and its primary and secondary unimolecular decomposition products. J Phys Chem A. 2018; 122: 4920- 4934.

16 Cocker W, Hanna DP, Shannon PVR. The chemistry of terpenes. Part VI. Pyrolysis and acid-catalysed rearrangements of (+)-car-3-ene and some derivatives. J Chem Soc, C. 1968: 489- 495.

17 Sharath N, Chakravarty H, Reddy K, Barhai P, Arunan E. Pyrolysis of 3-carene: experiment, theory and modeling. J Chem Sci. 2015; 127: 2119- 2135.

18 Van de Vijver R, Zádor J. KinBot: automated stationary point search on potential energy surfaces. Comput Phys Commun. 2020; 248:106947.

19 Vandewiele NM, Van Geem KM, Reyniers M-F, Marin GB. Genesys: kinetic model construction using chemo-informatics. Chem Eng J. 2012; 207-208: 526- 538.

20 Van de Vijver R, Vandewiele NM, Vandeputte AG, et al. Rule-based ab initio kinetic model for alkyl sulfide pyrolysis. Chem Eng J. 2015; 278: 385- 393.

21 Herbinet O, Battin-Leclerc F. Progress in understanding low-temperature organic compound oxidation using a jet-stirred reactor. Int J Chem Kinet. 2014; 46: 619- 639. 
22 Namysl S, Pelucchi M, Herbinet 0, Frassoldati A, Faravelli T, Battin-Leclerc F. A first evaluation of butanoic and pentanoic acid oxidation kinetics. Chem Eng J. 2019; 373: 973- 984.

23 Montgomery JA, Frisch MJ, Ochterski JW, Petersson GA. A complete basis set model chemistry. VI. Use of density functional geometries and frequencies. J Chem Phys. 1999; 110: 2822- 2827.

24 Van de Vijver R, Van Geem KM, Marin GB. On-the-fly ab initio calculations toward accurate rate coefficients. Proc Combust Inst. 2019; 37: 283- 290.

25 Frisch MJ, Trucks GW, Schlegel HB, et al. Gaussian 16. Wallingford, CT, 2016.

26 Kee RJ, Rupley FM, Miller JA, et al. Reaction Design, Inc. San Diego, CA, 2010.

27 Sabbe MK, Saeys M, Reyniers M-F, Marin GB, Van Speybroeck V, Waroquier M. Group additive values for the gas phase standard enthalpy of formation of hydrocarbons and hydrocarbon radicals. J Phys Chem A. 2005; 109: 7466- 7480.

28 Benson SW, Buss JH. Additivity rules for the estimation of molecular properties. Thermodynamic properties. J Chem Phys. 1958; 29: 546- 572.

29 Lay TH, Bozzelli JW, Dean AM, Ritter ER. Hydrogen atom bond increments for calculation of thermodynamic properties of hydrocarbon radical species. J Phys Chem. 1995; 99: 14514- 14527.

30 Cohen N. Revised group additivity values for enthalpies of formation (at $298 \mathrm{~K}$ ) of carbon-hydrogen and carbon-hydrogen-oxygen compounds. J Phys Chem, Ref Data. 1996; 25: 1411- 1481.

31 Sabbe MK, De Vleeschouwer F, Reyniers M-F, Waroquier M, Marin GB. First principles based group additive values for the gas phase standard entropy and heat capacity of hydrocarbons and hydrocarbon radicals. J Phys Chem A. 2008; 112: 12235- 12251.

32 Saeys M, Reyniers M-F, Marin GB, Van Speybroeck V, Waroquier M. Ab initio group contribution method for activation energies for radical additions. AIChE J. 2004; 50: 426- 444.

33 Sabbe MK, Reyniers M-F, Van Speybroeck V, Waroquier M, Marin GB. Carbon-centered radical addition and beta-scission reactions: modeling of activation energies and pre-exponential factors. Chem Phys Chem. 2008; 9: 124- 140.

34 Vandeputte AG, Sabbe MK, Reyniers M-F, Van Speybroeck V, Waroquier M, Marin GB. Theoretical study of the thermodynamics and kinetics of hydrogen abstractions from hydrocarbons. J Phys Chem A. 2007; 111: 11771 - 11786.

35 Saeys M, Reyniers M-F, Van Speybroeck V, Waroquier M, Marin GB. Ab initio group contribution method for activation energies of hydrogen abstraction reactions. Chem Phys Chem. 2006; 7: 188- 199.

36 Sabbe MK, Vandeputte AG, Reyniers M-F, Waroquier M, Marin GB. Modeling the influence of resonance stabilization on the kinetics of hydrogen abstractions. PCCP. 2010; 12: 1278- 1298.

37 Van de Vijver R, Sabbe MK, Reyniers M-F, Van Geem KM, Marin GB. Ab initio derived group additivity model for intramolecular hydrogen abstraction reactions. PCCP. 2018; 20: 10877- 10894.

38 Sabbe MK, Reyniers M-F, Waroquier M, Marin GB. Hydrogen radical additions to unsaturated hydrocarbons and the reverse beta-scission reactions: modeling of activation energies and pre-exponential factors. Chem Phys Chem. 2010; 11: 195- 210. 